\title{
COVID-19 AND ISCHEMIC HEART DISEASE EMERGENCIES: WHAT CARDIAC SURGERY SHOULD EXPECT?
}

\author{
Emanuele Pilato ${ }^{1}$, Rachele Manzo ${ }^{1}$, and Giuseppe Comentale ${ }^{2}$ \\ ${ }^{1}$ Università degli Studi di Napoli Federico II Scuola di Medicina e Chirurgia \\ ${ }^{2}$ Universita degli Studi di Napoli Federico II
}

April 28, 2020

\begin{abstract}
The incidence of mechanical complications of acute coronary syndromes (ACS) needing cardiac surgery has reduced significantly in the last years due to early diagnosis and treatments. Covid-19 pandemic, however, would generate in the patients a sense of fear regarding access to the ERs so they probably underestimate symptoms such as chest pain or angina equivalents until situation does not became critical. In this way, this behaviour could create a vast pool of patients who will enter the hospital in much more critical situations and with mechanical complications of an evolving ACS needing cardiac surgery treatment.
\end{abstract}

\section{Covid-19 and Ischemic Heart Disease Emergencies: What Cardiac Surgery should expect?}

Running title: Cardiac surgery After covid 19

Pilato Emanuele, $\mathrm{MD}^{1}$; Manzo Rachele, $\mathrm{MD}^{1}$; Comentale Giuseppe, $\mathrm{MD}^{1}$

${ }^{1}$ Division of Cardiac Surgery, "Federico II" University of Napoli (Italy)

\section{For Correspondence}

Comentale Giuseppe, MD

giuseppe.comentale@unina.it,Division of Cardiac Surgery

"Federico II" University - Via Sergio Pansini n5, 80131 - Napoli (Italy)

Main Body

Back to the past? The incidence of mechanical complications of acute coronary syndromes (ACS) needing cardiac surgery has reduced significantly in the last years due to early diagnosis and treatments. Primary coronary angioplasty of culprit lesions, indeed, allowed rescue of vital myocardium after ACS with significant impact on the functional recovery of the heart and long-term morbidity.

COVID-19 pandemic may oddly lead to a changing scenario and significantly modify the cardiac surgery population that we will be dealing with in the next weeks. The widespread demand for intensive and sub-intensive care beds, as well as a cardiac tropism of SARS-COV-2 [1], has brought to an extensive reassessment of hospitals and emergency rooms (ERs) into COVID-dedicated structures, making them potential contagion areas. In Italy, for example, more than 10\% of COVID-patients are healthcare providers showing how a severe protection policy among hospitals staff is mandatory to protect not only themselves but all kinds of patients. In support of this, "Cotugno" Infectivological Hospital of Napoli, through the highest protection/decontamination protocols, is the only Italian hospital without healthcare SARS-COV-2 infections. 
This phenomenon, therefore, would generate in the patients a sense of fear regarding access to the ERs: the patient with an ACS, indeed, especially diabetic ones, probably ends up underestimating symptoms such as chest pain or angina equivalents and not going to the ER quickly, relying on home remedies. This behaviour, contrary to what would be expected given the current predilection of an endovascular treatment of ACSs aimed at minimizing the impact on intensive care [2], could frustrate the prevention strategies based on the earliness of intervention implemented in these years and thus creating a vast pool of patients who will enter the hospital in more critical situations and with mechanical complications of an evolving ACS. Papillary muscles ruptures and acute mitral regurgitations, ruptures of the interventricular septum or of the walls of the heart, in fact, together with the aneurysmal dilations of untreated necrotic areas could became an important part of the cardiac surgery population in the next weeks.

For this purpose, strategies concerning people education and a centralization of cardiovascular care towards "protected" structures could restore the patient's confidence in going to the ERs at an early stage of symptoms and stem a phenomenon that could trigger an important increase in cardiovascular morbidity and mortality.

\section{References}

1. Fried JA et al. The Variety of Cardiovascular Presentations of COVID-19. Circulation. 2020 Apr 3.

2. Tarantini G et al. Italian Society of Interventional Cardiology (GISE) Position Paper for Cath labspecific Preparedness Recommendations for Healthcare providers in case of suspected, probable or confirmed cases of COVID-19. Catheter Cardiovasc Interv. 2020 Mar 29. 